\title{
Development and Implementation of Cost-effective Flight Simulator Technologies
}

\author{
Brent Cameron, Hooman Rajaee, Bradley Jung, Robert Langlois \\ Carleton University \\ 1125 Colonel By Dr., Ottawa, Canada \\ Brent.Cameron@carleton.ca; Hooman.Rajaee@carleton.ca; \\ Bradley.Jung@carleton.ca; Robert.Langlois@ carleton.ca
}

\begin{abstract}
Low cost visual, audio, vibration, and control loading cueing technologies were implemented on an initially-obsolete flight simulation training device by Vector Training Systems. This resulted in the Carleton University Redeveloped Vector Simulator (CURVS). $\mathrm{X}$-Plane 10 was used as the new simulation environment and appropriate data acquisition hardware and software was used to control mechanical instruments and pilot controls. An ultra-wide-angle triple-channel HD cylindrical projection system was built to replace the original low resolution $(800 \times 600$ pixels $)$ single-channel system. The screen was constructed out of steel tubing and PVC screen fabric. The images from three projectors were blended together to produce a seamless, asymmetric $220^{\circ}$ field of view offset to the pilot's side, an innovative feature which gives superior situational awareness to a student pilot practising landing manoeuvres. Vibration cueing was implemented with a seat-mounted vibration transducer driven by a custom engine audio recording acquired from a Cessna 172 aircraft. Control loading was also implemented. This feature increases the realism of the pilot experience by allowing the pilot to become accustomed to feeling the resistive forces from the controls during various manoeuvres. Test subjects noted very satisfactory experiences with CURVS. Control loading and ultra-wide-angle projection systems are invaluable elements to flight training, and greatly increase transfer of training between the simulator and an actual aircraft. However, these technologies are typically only available on expensive, high-end simulators. The innovative, cost effective control-loading and projection technologies implemented in this project will help bring these critical features within reach of a much greater body of student pilots, and enable the creation of more realistic, cost effective training devices in general.
\end{abstract}

Keywords: Ab initio, audio cueing, control loading, flight simulator, flight training, vibratory cueing, visual cueing

\section{Introduction}

Full-flight simulators (FFSs) and flight simulation training devices (FSTDs) are available for large commercial-aircraft and are used for the training of commercial airline pilots. While these devices are used primarily to improve training effectiveness and safety at reduced cost, they also improve ab initio, or introductory, pilot training. However, devices suitable for ab initio flight training are either not available, lack fidelity, or are prohibitively expensive for many flight schools. The Carleton University Applied Dynamics Laboratory (ADL) has been developing ways to make high-fidelity flight simulators more accessible to small flight schools by developing a cost-effective hardware and software simulator architecture using commercial-off-the-shelf (COTS) components. The ADL developed these technologies starting with an obsolete, non-functional Vector Training Systems Cessna 172 flight simulator cockpit as a testbed. This simulator's software and hardware were no longer up to modern quality and reliability standards and the manufacturer has long since gone out of business. The ADL has prior experience implementing low-cost flight simulation technologies on its KatanaSim platform, where a Diamond DA-20 FSTD was created from a used DA-20 cockpit. In the KatanaSim, the instruments were replaced with simulated replicas, and the entire cockpit was placed on a six degrees of freedom motion platform for motion cueing.

The original Vector simulator ran rudimentary flight simulation software on a now obsolete Inmos Transputer and had an original price tag of about $\$ 300,000 \mathrm{CAD}$ (2016). The ADL has replaced this system with a set of four modern, high-performance gaming-grade PCs and graphics cards. They run X-Plane 10, a commercially-available flight simulator program that uses blade element theory for the flight model and whose internal variables are accessible via an application-programming interface (API). Originally, the pilot's view was restricted through carefully-placed cockpit window cutouts, such that the pilot was only able to see a small, rectangular projection screen ahead. Peripheral vision was afforded by a selector knob in the cockpit which could rotate the projected view to one side or the other. Peripheral and over-the-shoulder vision is necessary in an aircraft for accurate situational awareness. However, most existing simulators do not provide a horizontal field of view much greater than $200^{\circ}$ [1]. With limited over-the-shoulder visuals, most current simulators instead rely on the instructor to cue the student, as key visual landmarks, such as runways, are not visible during much of the pre-landing manoeuvring sequence performed by general aviation aircraft. This reduces transfer of training and realism. Existing commercial cylindrical projection and screen coating solutions are prohibitively expensive; however, similar end results can be obtained using modern, cost-effective high-definition home theatre projectors, PVC screen material, and commonly-available construction products such as schedule 40 steel pipe and structural clamps (e.g., the Kee Klamp line of products [2]). Using these materials, the ADL implemented a cost-effective, ultra-wide-angle projection system with an asymmetric horizontal field of view of approximately $220^{\circ}$, thereby enabling the over-the-shoulder view.

A cost-effective control-loading methodology was also implemented which enables the student pilot to become accustomed to the yoke and pedal forces encountered during the various stages of flight, a feature which is not typically available on existing entrylevel flight training devices. While the original simulator did have control loading, the motors needed to be interfaced with the new 
simulation software. This was accomplished through commercially-available LabJack data acquisition devices coupled with custom interface software [3].

Audio recordings were sampled from live flight tests to replace the generic aircraft engine audio from X-Plane. These recordings were also used as the input signal for seat shaker transducers to enable vibratory cueing. Seat shakers are typically not used on entry-level simulators but offer the pilot valuable cues regarding engine RPM during manoeuvres and also help to indicate the transition from runway to flight and vice versa.

The resulting FSTD has since been re-branded as the Carleton University Redeveloped Vector Simulator (CURVS). These simulation technologies offer the potential for widespread improvement in the quality, fidelity, and cost-effectiveness of ab initio flight training, as well as corresponding improvements to aviation safety.

CURVS will be used by the Waterloo Wellington Flight Centre (WWFC) to conduct ab initio pilot training, as well as supporting instrument flight training and re-certification. It will be certifiable by Transport Canada as a Flight Training Device to Level $2+$ status. This will allow time spent in the simulator to be logged as equivalent time in an actual aircraft. Simulators typically cost significantly less per hour to operate compared to an actual aircraft, provide a demonstrated headstart to ab initio pilot training, and significantly reduce the air time required for a student pilot to reach the proficiency required to fly solo [4]. Most importantly to the authors, use of the CURVS simulator in actual pilot training will provide valuable feedback on the performance of the newly-developed technologies in real-world usage scenarios.

\section{Redevelopment of Cockpit Interface System}

The original electronic interface and control boards used in the simulator were custom-made for the device, and as the original manufacturer in Austria was found to have gone out of business and documentation was unavailable, it was decided that a new, costeffective interface and control system be designed and implemented. A number of modern, low-cost interface solutions were considered, including the Arduino microcontroller, Raspberry Pi, and BeagleBone Black single-board Linux computers. In the end, the LabJack T7 data acquisition (DAQ) and control device was selected as the core of the new system. This device was selected for its robustness, simplicity of use, $\mathrm{C}++/ \mathrm{C}$ programming interfaces, voltage tolerances, excellent documentation, ability to communicate via an Ethernet connection, and relatively low cost per unit (\$399 USD in 2015). The redesigned cockpit interface system is shown in Figure 1.

\section{Reverse Engineering}

The original simulator was received from our industrial partner in a non-functional state, with some printed operating instructions available but no technical documentation such as wiring diagrams or schematics. The simulator was examined, and while the original simulation software appeared to be corrupted, much of the hardware in the cockpit was still functional. Software backups were unavailable, so rather than attempt to restore the simulator to its original state (circa 2002), seen in Figure 2 and 3 , it was decided to keep as many of the cockpit components as possible, interfacing them to modern flight simulation and image generation PCs. Copies of some of the original technical documentation were later obtained from both a former employee of the manufacturer, and an American technical college which still maintains two examples of a similar simulator by the same manufacturer.

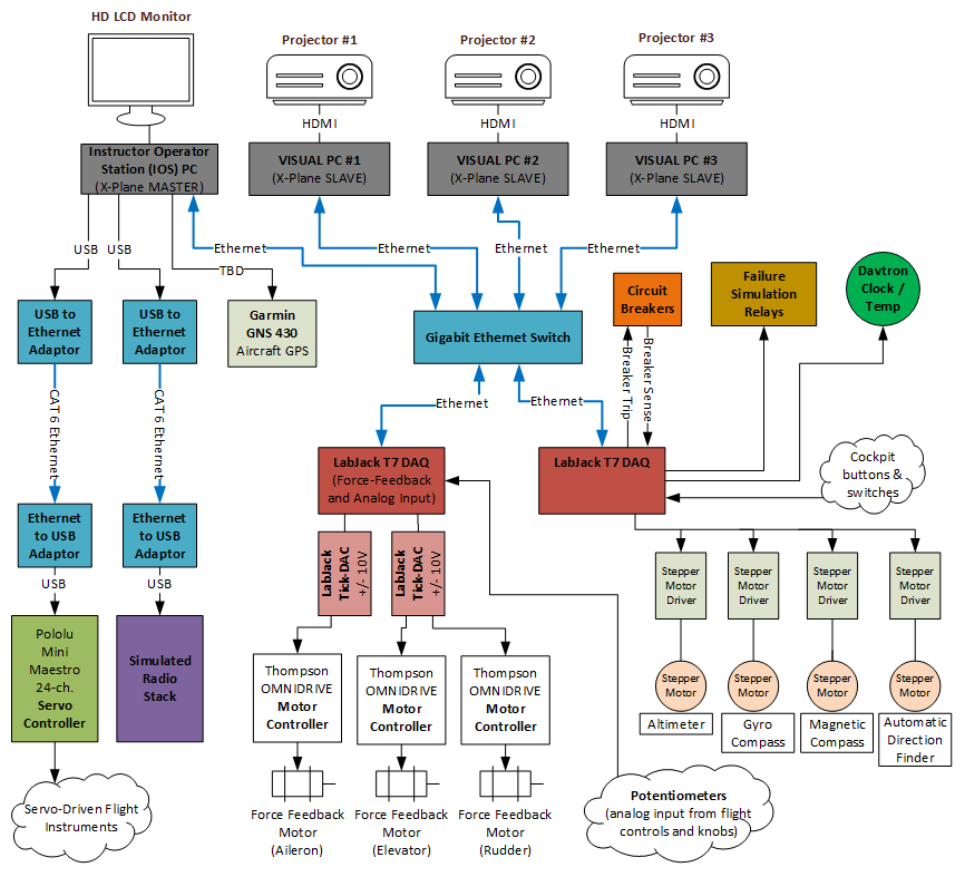

Fig. 1: CURVS system architecture. 


\subsection{Simulated Flight Instruments}

The original flight instruments, examples of which are seen in Figures 4 and 5, consisted of both servomotor and stepper motorbased units. In total, there were 18 servomotors and 4 stepper motors to be interfaced to and controlled. The servomotors were found to be standard Futaba-brand units, such as are commonly used in remote-control model aircraft. The width of the pulses fed to the servomotors on their signal lines determine the angular positions to which they turn. Due to the large number of motors requiring simultaneous control, a dedicated servo-control board (Pololu Mini Maestro) was selected [5]. This is a low-cost device intended for hobby robotics applications (\$49.95 USD), and includes a C\# software development kit (SDK).

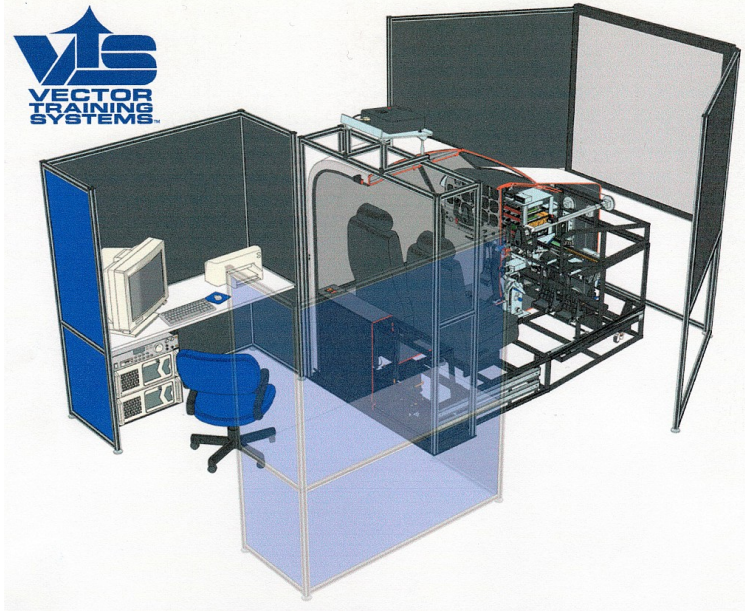

Fig. 2: VectorSim rendering.

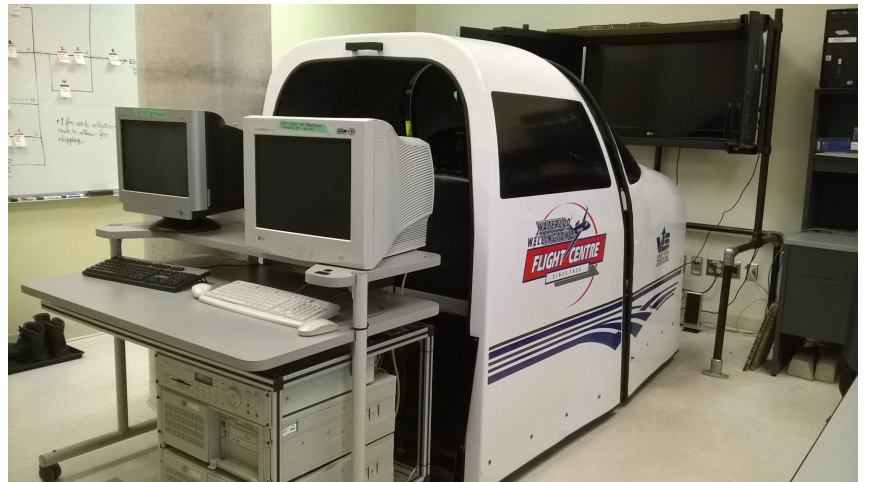

Fig. 3: VectorSim in the ADL.

Due to the need for continuous rotation, the magnetic compass, directional gyro, altimeter, and automatic direction-finder instruments all utilized stepper motors in their construction. All of the original instruments were found to be extremely well made, featuring tough plastics, aluminum plates, and brass gears. Since stepper motors provide no position feedback, a combination of slotted disc, infrared LED, and photodetector are used to determine a predefined zero position for each instrument. Upon initialization, the stepper motor is rotated until light is detected though the slotted disc. This initial position is then incremented or decremented as appropriate depending on the current state of the simulation. The original stepper motor driver chips have been identified and located, and are available for reuse.

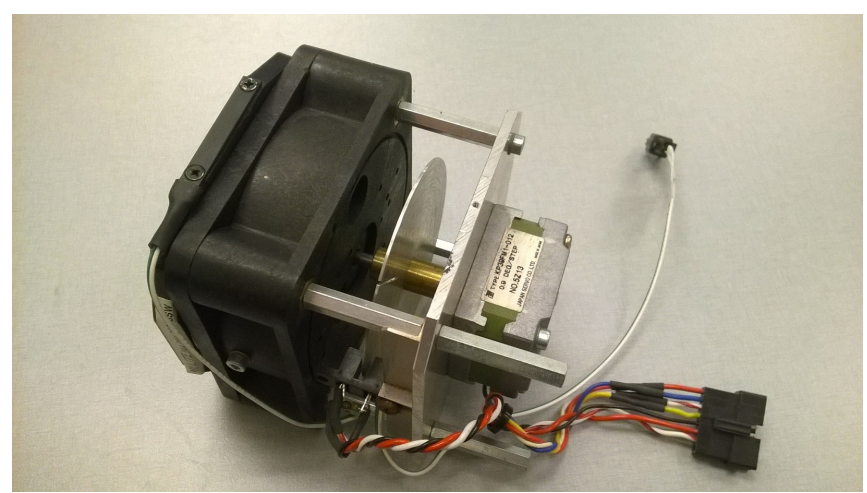

Fig. 4: Stepper motor instrument - automatic direction finder.

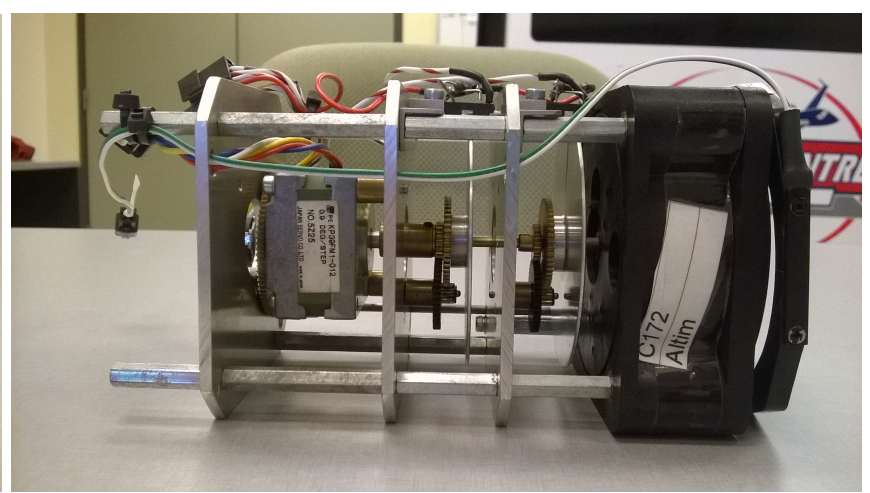

Fig. 5: Stepper motor instrument-altimeter.

\subsection{Flight Instrument Software Interface}

As of this writing, the software interface between the servo-driven flight instruments and X-Plane is a console application written in C\#. C\# was selected primarily due to its use by the Pololu servo controller SDK; however C\# also includes a number of convenient built-in functions for receiving data packets from X-Plane using the user datagram protocol (UDP). This approach currently requires that the correct datasets be manually selected for output from within X-Plane. X-Plane will then transmit a UDP packet at the specified rate (currently $60 \mathrm{~Hz}$ ), containing each of the selected datasets in ascending numerical order. This approach is susceptible to error, however, as the ordering of the datasets in each packet is dependent on the total number of datasets selected. These settings are easily accessible to the end user from within X-Plane. A better approach would be to parse each datagram based on dataset number instead of position within the datagram. This would add additional computational complexity to the $\mathrm{C} \#$ interface program; however, the resulting increase 
in latency would likely be slight. This would greatly increase reliability of the simulator and prevent an accidental click by the user from breaking the simulation.

It is anticipated that the stepper motor instruments will be controlled using the LabJack DAQ, as this device supports a number of pulse-capable outputs which eliminate the need to manually toggle digital output pins on and off for each rotation step required. In addition, pilot inputs to the instruments, such as the barometric pressure setting for the altimeter, will be read by the DAQ.

\subsection{Control Loading System}

The primary flight controls of a typical small aircraft consist of both a yoke (or stick) and rudder pedals. The force required to deflect a flight control by a given amount is directly proportional to the square of the airspeed [6][4]. The squared relationship is due to dynamic pressure, which is defined as the additional pressure experienced by an object due to its velocity. Dynamic pressure, $q$, is defined mathematically as follows:

$$
q=\frac{1}{2} \rho v^{2}
$$

where $\rho$ is the density of the air and $v$ is the airspeed [7]. The original control-loading system consisted of three industrial brushless servomotors, one for each axis of control (pitch, roll, and yaw). Each motor was connected to the flight controls via a system of linkages which ran under the floor of the simulator. Each motor controller was rated for $500 \mathrm{~W}$ continuous output power. Although support was not available from the manufacturer due to the age of the product, the original motor controller manual was located online [8]. This manual was used in conjunction with original documentation from a similar simulator to identify the appropriate junction strip within the simulator, and reverse-engineer the custom pinout used to interface to the motor controllers. The motors were tested by applying a $+/-10$ $\mathrm{V}$ analogue signal across the command inputs of each motor controller, and all were found to be fully functional.

The torque output by the control loading motors is directly proportional to the analogue voltage applied between the CMND+ and CMND- terminals of the motor controllers. However, the resultant force felt by the pilot on the controls depends on the mechanical advantage provided by the linkages connecting the motors to the controls.

Engineering drawings for the system of linkages between the control loading motors and the pilot's controls were unavailable. Thus, it was decided that the most expedient and accurate way to quantify the force output to the pilot for a given voltage applied to the motor controller was to measure the forces directly with a scale. An example calibration curve is shown in Figure 6 . The curves were found to be essentially linear, with a small dead-zone around zero volts, which was assumed to be caused by friction in the linkages.

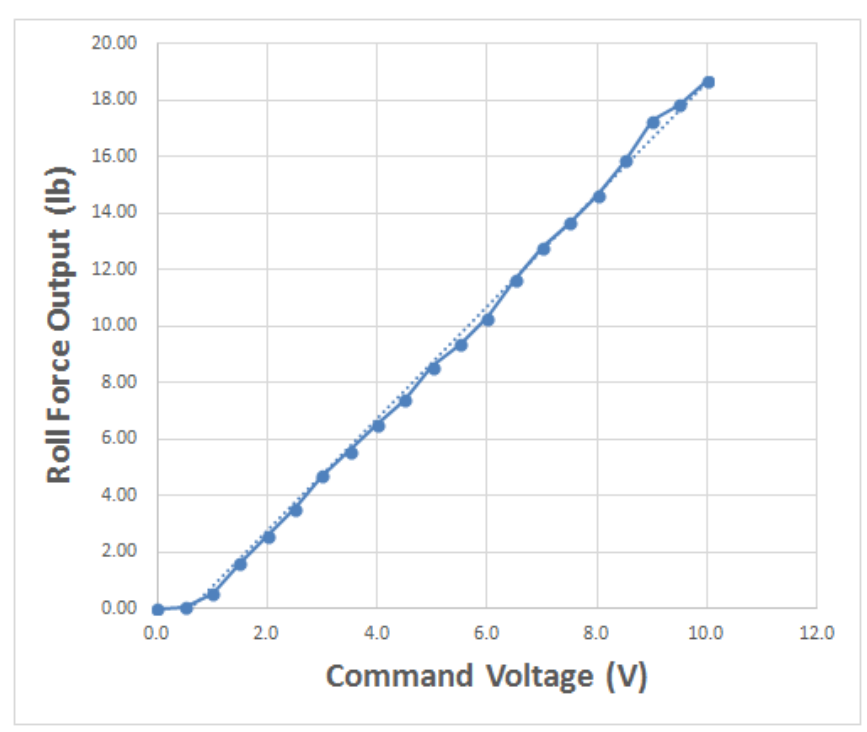

Fig. 6: Roll force verses input voltage.

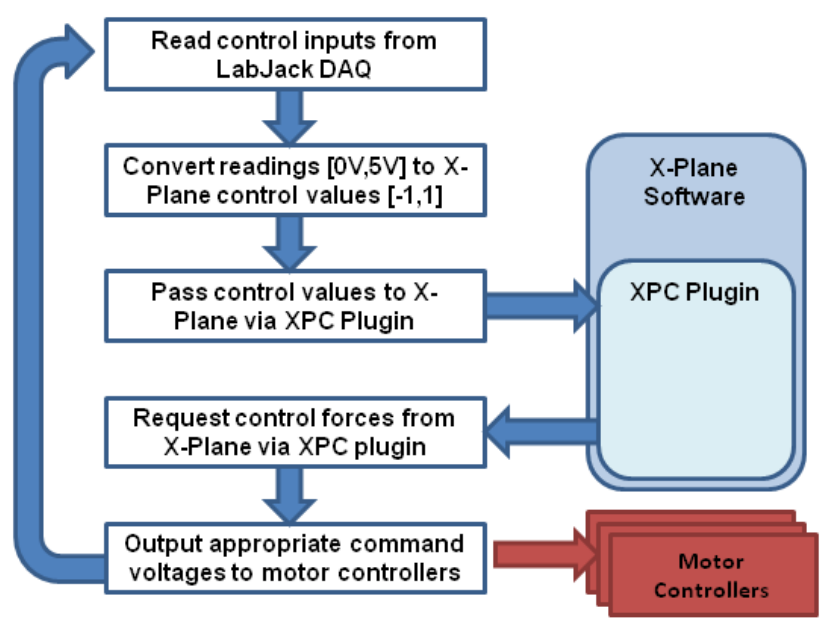

Fig. 7: Fundamental logic of the control loading interface program.

\subsection{Control Loading Software Interface}

In contrast to the flight instruments, the control loading interface program was written in $\mathrm{C}++$. This allowed communication between X-Plane and the control loading motors to be handled by the open-source X-Plane Connect (XPC) plugin available from NASA [9]. Use of this plugin and the accompanying C++ functions to handle UDP communication with X-Plane allowed development time to be focused on implementing simulator functionality, as opposed to dealing with the intricacies of byte-level C++ network communication.

The position of the flight controls is measured by linear potentiometers. The voltage values produced are then digitized by a LabJack T7 DAQ. Since calculation of control forces requires that the instantaneous position of the flight controls be known, the control loading interface program is also responsible for sensing control positions and passing them to X-Plane. X-Plane then generates appropriate control forces based on its own closed-source logic. The control loading program takes the form of an infinite loop, whose fundamental logic can be seen in Figure 7. 


\subsection{Control Loading System Stability}

At higher than normal airspeeds (i.e., above cruise speed), it was found that the control loading system could become unstable, with the controls tending to oscillate back and forth instead of settling back to their neutral position. Lubricating the relatively-stiff control linkages was found to exacerbate the oscillation problem, as frictional damping was thereby reduced. Limiting the rudder force from within X-Plane reduced the tendency of the rudder pedals to oscillate. However, this also reduced the fidelity of the simulation compared with the actual aircraft. Increasing the internal X-Plane damping coefficient was found to improve the stability of the yoke, but was not able to completely eliminate oscillation of the rudder pedals. In fact, for higher values of the rudder damping coefficient, the rudder pedals became coarse-feeling and hard to move, as if even pilot input was being damped.

\subsection{Hypothesized Causes for Control Loading Instability}

Instabilities in the control loading system may be due to: (a) lack of damping; and (b) a relatively-large simulation cycle timestep. First, with no physical dampers in the control loading system, only the relatively-low retarding force of friction is present to reduce the deflection rate of the controls to zero as they approach the neutral position. If the deflection rate is not reduced to zero by the time the neutral position is reached, an overshoot will result, thus setting the stage for a subsequent overshoot in the opposite direction. Second, the control loading interface program currently operates in lockstep with the X-Plane framerate $(60 \mathrm{~Hz})$. Running the control loading program at a higher rate would allow the control forces to be reduced more quickly as the controls re-centre. By Newton's second law, acceleration is a direct result of applied force. With no physical damping and minimal friction, the controls are therefore under continuous (although decreasing) acceleration until applied control force becomes zero at the neutral point. Being able to reduce control force more quickly in response to high deflection rates will reduce the tendency to overshoot. For this reason, many commercial control loading systems operate with much higher update frequencies, e.g. $500 \mathrm{~Hz}$ in the case described in Reference [10].

\subsection{Proposed Solutions for Control Loading Instability}

First, it is proposed that artificial damping be implemented in the control loading interface program in addition to (or instead of) the damping functionality provided within X-Plane. In order to prevent the system from attempting to damp out the pilot's own inputs to the controls, the artificial damping should only activate above a certain threshold control deflection rate. This should damp out the current oscillatory tendencies of the controls for large, sudden control displacements at high airspeeds, without interfering with normal movement of the controls. Second, if artificial, rate-triggered damping is insufficient to stabilize the controls for all regions of the flight simulation envelope, increasing the update rate of the control loading interface program should be investigated.

\section{Cueing Systems}

In the CURVS project, three main cueing systems were designed, implemented, and tested: visual cueing, auditory cueing, and vibratory cueing. The visual cueing system was a major upgrade to enhance the FSTD's display to a high-fidelity wrap-around system cost-effectively using commercial-off-the-shelf (COTS) components. The auditory cueing system is also realistic and uses modified actual aircraft recorded sounds. The vibratory cueing system was introduced to induce vibrations in the pilot and co-pilot seats that replicate the vibrations experienced in the aircraft during various manoeuvres. All the cueing systems were passed through the phases of design, development, and analysis. Quantitative and qualitative tests were performed to assess CURVS's cueing systems responses. Quantitative data was recorded both from the actual aircraft and the simulator in flight tests for comparison. Frequency analyses were performed on the data to evaluate the accuracy of the simulator responses. Finally, qualitative tests were performed to collect feedback from pilots. The cost of the audio, visual, and vibratory cueing systems amounted to about \$14,000 CAD.

The visual cueing system of CURVS is an asymmetric $220^{\circ}$ cylindrical wrap-around projection system. The screen consists of a $180^{\circ}$ field of view (FOV) in front of the pilot with an extra $40^{\circ}$ FOV on the left rear side as illustrated in Figure 8 . This type of display enables the pilot to look over his/her shoulder to see the runway while flying in a standard left-handed circuit. This feature is very beneficial for ab initio training in which pilots learn how to take-off and land the aircraft in a left-handed circuit.

\subsection{Visual Cueing}

The screen support structure was designed and a finite element analysis was performed using Creo Parametric with a safety factor of 2 against yield. The frame was built using steel tubes that were accurately bent to the desired radius by a computer numerical control (CNC) bending machine. Also, PVC fabric material, having a reflective gain factor of 1.1, suitable for use in a dark room, was attached to the frame [11]. Three short-throw projectors were chosen to generate the simulation image on the screen such that their images were soft-edge blended and their geometrical distortions were corrected using a COTS software package called Immersive Display Pro [12]. The hardware of the image generator consisted of four high-performance COTS PCs, three of which were used for generating the visual channels while the fourth was used to run the flight model in response to pilot inputs. The image generation capability that is intrinsic to $\mathrm{X}$-Plane 10 was used as it is both effective and COTS. The complete setup is shown in Figure 9. Figure 10 shows the screen and projector setup, as well as the soft-edge blending.

The hardware architecture of the PCs was chosen such that there was a balance between cost-effectiveness and performance. Regarding computer speed, each must be able to run X-Plane at 60 frames per second to generate a smooth sense of flying. This was important as highly-detailed custom scenery was embedded into the simulator for the Waterloo Airport (for WWFC). The more detailed the scenery contents, the faster the PCs must be. Therefore, the trade-off between the two was chosen meticulously.

The custom scenery consisted of three different layers on top of each other: first, the terrain data that comes with X-Plane modified with satellite imagery draped upon it and placed manually; second, the location of roads, rivers, lakes, houses, etc that had to be defined for the generic object libraries within X-Plane and is called overlay scenery; and third, the airport and custom scenery content on top of all the other layers. The coordinates of the overlay scenery were exploited from the Open Street Map (OSM) database and other sources by using a software package called HD Mesh Scenery [13]. The Waterloo airport scenery was completely custom built using ortho-photos and custom-designed 3D objects such as hangars, buildings, facades, etc. that were designed using open-source 3D modelling software and were put together in X-Plane using World Editor software that is a free tool included in the X-Plane package [14]. All the necessary information was extracted from the Canada Flight Supplement, which is the official reference book for all Canadian airports [15]. The resulting scenery is shown in Figure 11. 

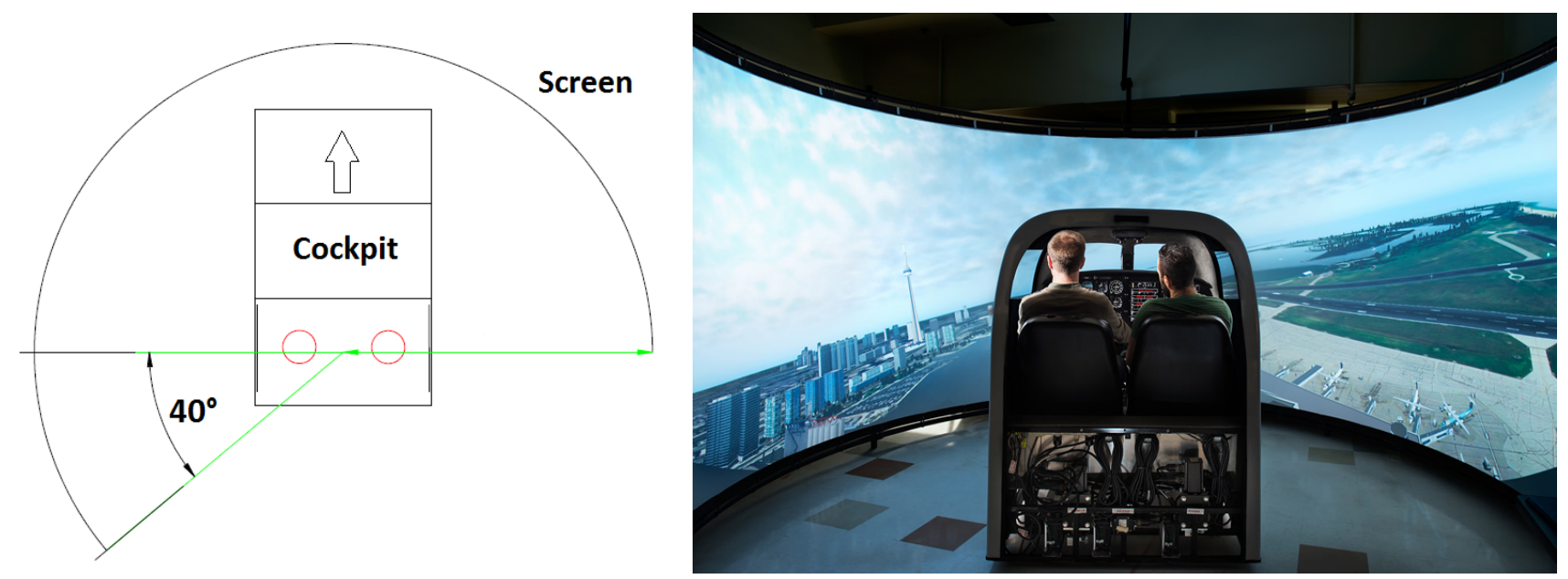

Fig. 8: The cylindrical screen with $220^{\circ}$ FOV.

Fig. 9: CURVS with new cylindrical screen.
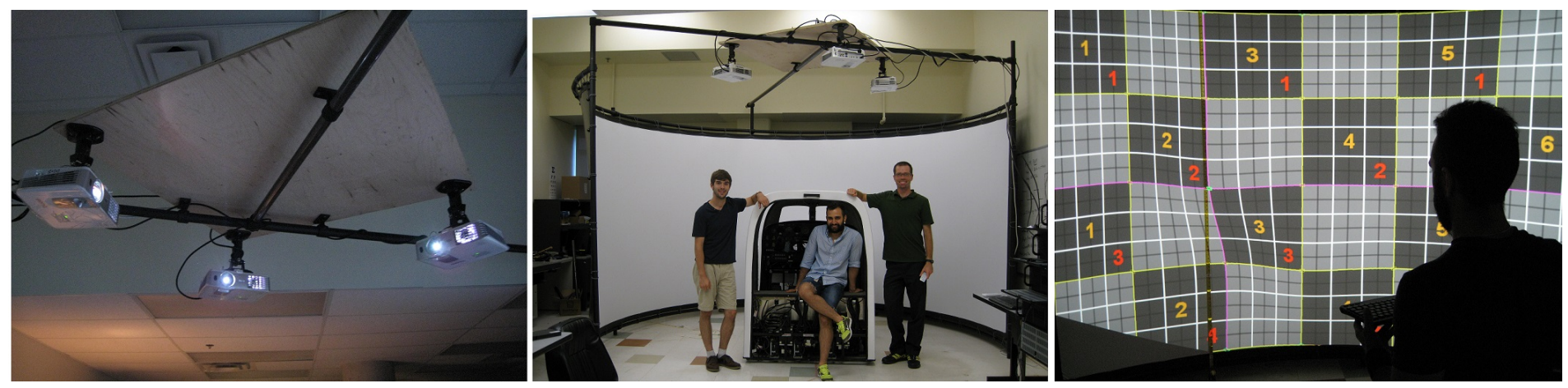

Fig. 10: The FSTD projection screen (left), final appearance (centre), and the soft-edge blending (right).

\subsection{Audio Cueing}

The audio cueing system employed a 5.1 channel amplifier and speaker system and a set of modified aircraft sound files that were recorded during aircraft flight tests. A stereo audio recorder was used to record sound files during different standard manoeuvres with various engine RPMs. The sound files were edited using an open-source software package and were then added to X-Plane. X-Plane has an internal algorithm that changes the pitch and volume of the sound signal based on engine RPM. The algorithm is not customizable; however, it's accuracy was tested. After recording the files and implementing them into the audio system, the same flight tests were performed in CURVS using the sound recorder and a decibel meter to evaluate the simulator sound system.

The collected data were also used for quantitative comparison of the simulator and the aircraft to measure how close the simulator sound system replicated the response of the aircraft. The results are shown in the form of power spectral density (PSD) plots in the results section.

\subsection{Vibratory Cueing}

The vibratory cueing in CURVS was a stand-alone cueing system. A ButtKicker-brand vibration transducer was employed to induce vibration in the seats as shown in Figure 14. The ButtKicker utilizes audio signals (that were recorded during flight tests) to generate vibration and uses an amplifier that filters out and amplifies the low-frequency components of the audio signals before sending them to the seat shaker unit. The seat shaker device is a cylindrical electromagnet that has a small piston inside which oscillates up and down based on the input signal fluctuations [16]. A portable accelerometer was utilized to collect acceleration data in the aircraft and the simulator during flight tests for comparison. The results are shown in the form of PSD plots in the quantitative test section.

\section{Results}

\subsection{Quantitative analysis}

A quantitative test was performed for both auditory and vibratory cueing systems where their behaviours were compared to the actual aircraft. The data for sound was in WAV file format which is a normalized data set and for the vibration in G forces. The Welch method was used to produce PSD plots for various manoeuvres. To generate vibration the recorded sound files are manipulated in X-Plane using its own built in algorithm to change the sound pitch. This signal is both used to generate sound (through speakers) and vibration (as 


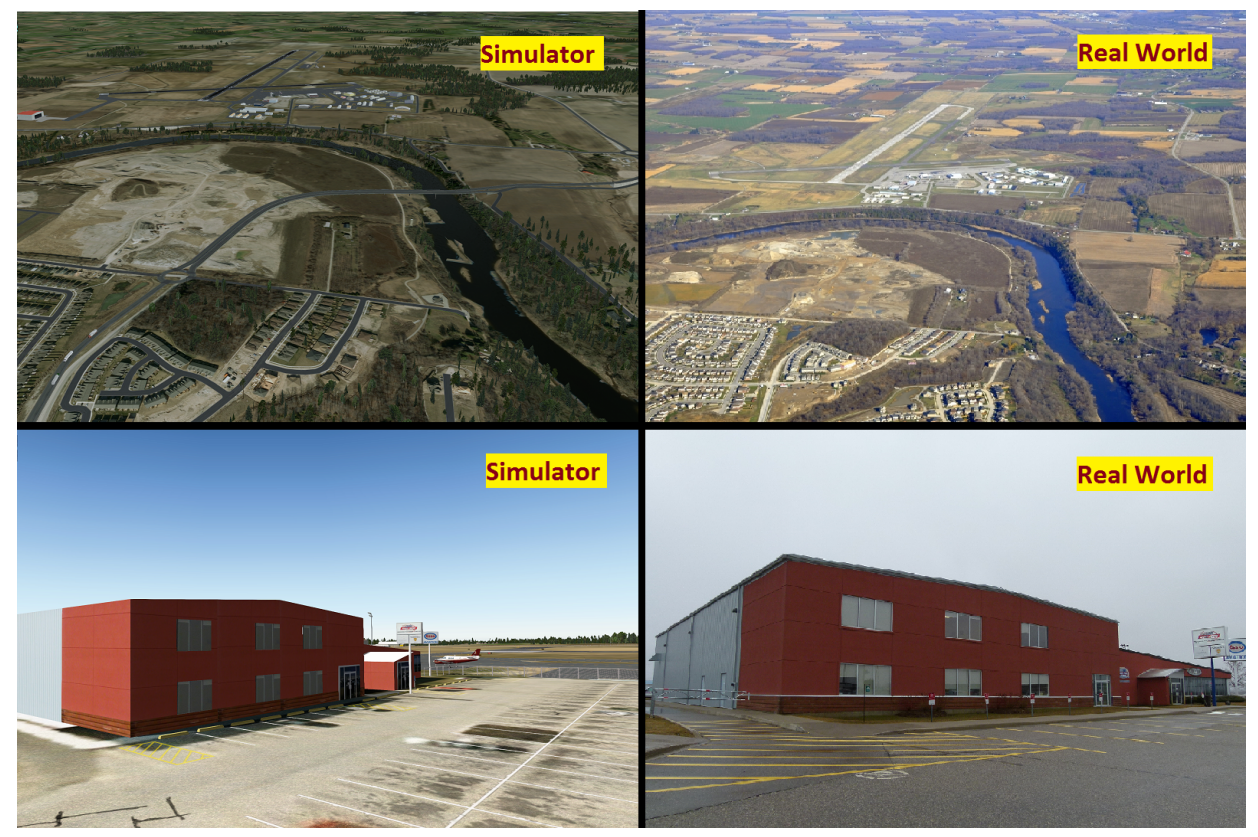

Fig. 11: The final custom scenery comparison.

input signals into the Buttkicker). Figure 12 illustrates the PSD analysis of the vibration at 1000 RPM while the aircraft was taxiing on the ground both in the simulator and in the aircraft. The PSD plot for vibration shows that the aircraft and the simulator response match in most of the frequencies. The highest and second highest peaks from the simulator are shifted about $10 \mathrm{~Hz}$ higher than the corresponding highest and second highest peaks from the aircraft PSD plot. Signals at these low frequency ranges are out of recording capability of the sound recorder and completely generated by X-Plane. Therefore any discrepancies are an artifact of the internal X-Plane model.

Figure 13 shows a comparison between the CURVS sound system and the actual Cessna 172 aircraft while the aircraft was cruising with the engine running at 2400 RPM. Signals below $45 \mathrm{~Hz}$ are not relevant because these frequencies are too low for both the sound generator and sound recorder. Although the simulator and aircraft curves do not perfectly match as well from $45-80 \mathrm{~Hz}$ the most significant peaks at $85 \mathrm{~Hz}, 130 \mathrm{~Hz}$, and $165 \mathrm{~Hz}$ directly match in this specific manoeuvre.
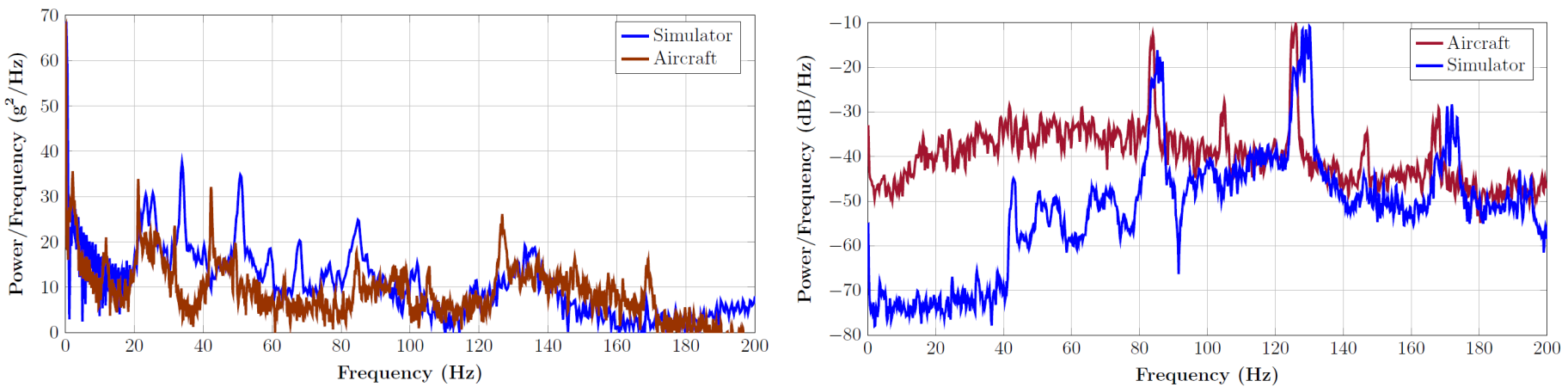

Fig. 12: PSD comparison of vibration while taxiing at 1000 RPM. Fig. 13: PSD comparison of sound while cruising at 2400 RPM.

\subsection{Qualitative Test}

A qualitative test was performed on CURVS by inviting eight pilots with various backgrounds and experience to evaluate the simulator. The pilots were asked to fly multiple circuits and give their opinions both verbally and written on a survey form. For the written feedback, the pilots had to fill out a questionnaire that was adapted from a prior NASA simulator test [17]. The questionnaire included rating questions as well as yes/no responses where some of the questions were targeting the comparison of the CURVS with the actual aircraft and some were comparing it with other existing commercially-available simulators. The results of the test using the answers to the main questions are summarized in Figure 15.

The results shows that the pilots ranked the general experience of the simulator cueing systems relatively high. Test pilots were very satisfied with the FOV, screen resolution, and framerate. The sound system and the vibratory cueing were ranked between Good 
and Excellent which means they had a positive impact on the simulator experience that pilots had in comparison to the actual aircraft and other simulators on the market.

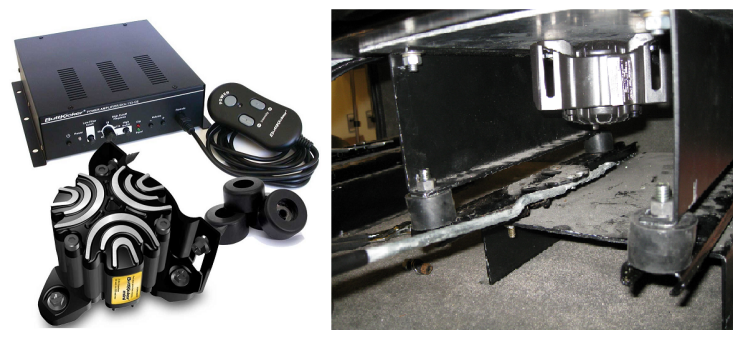

Fig. 14: The Left: ButtKicker simulation kit. Right: ButtKicker installed
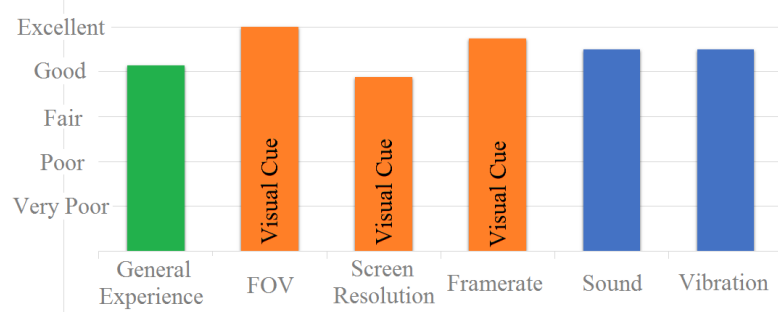

Fig. 15: Questionnaire result bar chart.

\section{Conclusion}

The ADL has designed and implemented a number of cost-effective technologies applicable for use in both FFSs and FSTDs. Using these technologies, an initially-obsolete and non-functional FFTD was redeveloped, modernized, and will be returned to service. Use of commercial off-the-shelf hardware and software was emphasized in order to minimize costs and increase maintainability in the long term. The specific technologies developed include a robust cockpit interface system for acquiring pilot input and driving mechanical flight instruments, a cost-effective control loading interface system for driving the legacy motor controllers already available, an innovative, $220^{\circ}$ asymmetric wraparound cylindrical projection system, highly-detailed 3D custom scenery, a 5.1-channel sound system paired with high-fidelity custom cockpit audio recordings, and enhanced haptic feedback via the use of seat vibration transducers. The redeveloped control loading system was found to exhibit instability at higher airspeeds and is being redesigned, while the visual, audio, and vibratory cueing technologies were evaluated by experienced pilots, who reported a high degree of satisfaction. This research will aid the development of a new generation of cost-effective, high-fidelity flight simulators and training devices, making the proven benefits of simulation in ab initio flight training more accessible to small flight schools.

\section{Acknowledgments}

The authors wish to express their thanks for the financial support of this research project by both Waterloo Wellington Flight Centre and the Ontario Centres of Excellence through the TalentEdge Internship Program. Special thanks are also due to Jon Roloff and Jared Wilhelmi for providing many pages of original simulator documentation. In addition, Harry Zanin of Flight Plus Software, Ltd. provided valuable advice regarding the development of the visual system, while Andras Fabian, of HD Mesh Scenery, graciously allowed the use of his software. Finally, Stefano Sandri was instrumental in the mechanical design and fabrication of the frame for the projection screen.

\section{References}

[1] Alsim. (2016). [Online]. Available: http://www.alsim.com/simulators/high-definition-visual-system/

[2] Kee Safety, Ltd. (2015, June). Kee Klamp Galvanized Steel Components. [Online]. Available: http://www.keesafety.ca/products/kee__klamp

[3] LabJack Corporation. (2016, February 25). T7 Datasheet. [Online.] Available: https://labjack.com/support/datasheets/t7.

[4] D. Alletron. Principles of Flight Simulation. American Institute of Aeronautics and Astronautics, VA, USA: Wiley, Reston, 2009.

[5] Pololu Corporation. (2016, February 29). Pololu Maestro Servo Controller Users Guide. [Online]. Available: https://www.pololu.com/docs/0J40/all

[6] I. Moir and A. Seabridge. Aircraft Systems: Mechanical, Electrical and Avionics Subsystems Integration. Chichester, West Sussex, UK: Wiley, 2008.

[7] J. D. Anderson, Fundamentals of Aerodynamics. New York, NY: McGraw-Hill, 2011.

[8] Thomson Motion Control Division. (2016, February 17). OMNIDRIVE Installation Manual for Models ODM-005 and ODM-005i. [Online]. Available: http://www.artisantg.com/info/P_djIzk.pdf

[9] J. Watkins C. Teubert. (2016, February 17). XPlaneConnect. [Online]. Available: https://github.com/nasa/XPlaneConnect

[10] BFF Design Ltd. (2016, February 17). BFF modular CL system brushless motor driver. [Online]. Available: http://bffsimulation.com/BFF-FFB-System.php

[11] Flexi White Material. (2015, July). [Online]. Available: http://www.carlofet.com/finished-edge-screens-with-grommets/flexiwhitefinished-edge-screens-with-grommets.html.VtTCwfkrKUk

[12] Fly Elise (2015, June). Immersive Display Pro. [Online]. Available: http://www.fly.elise-ng.net/

[13] A. Fabian. (2016, February 29). X-Plane 10 HD Mesh Scenery v3. [Online.] Available: http://www.alpilotx.net/downloads/x-plane10-hd-mesh-scenery-v3/

[14] WorldEditor. (2015, July). [Online]. Available:http://developer.X-plane.com/tools/worldeditor/

[15] Nav Canada, Canada Flight Supplement. Ottawa, Ontario, 2014.

[16] The Guitammer Company. (2016, March 16). ButtKicker. [Online.] Available: http://www.thebuttkicker.com/simulation-kit

[17] NASA: Benton L. Parris Anthony M. Cook, "Effects of visual and motion simulation cueing systems during takeoffs with engine failure," Technical report, NASA, 1978. 